\title{
Thermodynamic properties of binary hcp solution phases from special quasirandom structures
}

\author{
Dongwon Shin,* Raymundo Arróyave, and Zi-Kui Liu \\ Department of Materials Science and Engineering, The Pennsylvania State University, University Park, Pennsylvania 16802, USA
}

Axel Van de Walle

Engineering and Applied Science Division, California Institute of Technology, Pasadena, California 91125, USA

(Received 13 September 2005; revised manuscript received 25 April 2006; published 14 July 2006)

\begin{abstract}
Three different special quasirandom structures (SQS's) of the substitutional hcp $A_{1-x} B_{x}$ binary random solutions $(x=0.25,0.5$, and 0.75$)$ are presented. These structures are able to mimic the most important pair and multi-site correlation functions corresponding to perfectly random hcp solutions at those compositions. Due to the relatively small size of the generated structures, they can be used to calculate the properties of random hcp alloys via first-principles methods. The structures are relaxed in order to find their lowest energy configurations at each composition. In some cases, it was found that full relaxation resulted in complete loss of their parental symmetry as hcp so geometry optimizations in which no local relaxations are allowed were also performed. In general, the first-principles results for the seven binary systems (Cd-Mg, Mg-Zr, Al-Mg, Mo-Ru, Hf-Ti, Hf-Zr, and $\mathrm{Ti}-\mathrm{Zr}$ ) show good agreement with both formation enthalpy and lattice parameters measurements from experiments. It is concluded that the SQS's presented in this work can be widely used to study the behavior of random hep solutions.
\end{abstract}

DOI: 10.1103/PhysRevB.74.024204

PACS number(s): 61.66.Dk

\section{INTRODUCTION}

Thermodynamic modeling using the calculation of phase diagrams (CALPHAD) method ${ }^{1,2}$ attempts to describe the Gibbs energy of a system through empirical models whose parameters are fitted using experimental information. These descriptions allow the extrapolation of a system's thermodynamic properties to regions in the composition-temperature space that have not/cannot be accessed through experiments. These empirical models, however, are as good as the data used to fit them and are therefore limited by the availability of accurate experimental data. This limitation can be overcome by using theoretical calculations based on firstprinciples methods, which are capable of predicting the physical properties of phases with no experimental input. ${ }^{3}$ Unfortunately, despite their predictive nature, these methods are not yet able to calculate the thermochemistry of materials-especially multicomponent, multiphase systems - with the precision required in industry.

A natural way of improving the predictive capabilities of empirical models while maintaining their applicability to practical problems is by combining first-principles and CALPHAD techniques. Thanks to efficient schemes for implementing density functional theory (DFT),${ }^{4}$ the almostroutine use of first-principles results within the CALPHAD methodology has become a reality. In this hybrid approach, the energetics obtained through electronic structure calculations are used as input data within the CALPHAD formalism to obtain the parameters that describe the Gibbs energy of the system. ${ }^{5}$

The first-principles electronic structure calculations of perfectly ordered periodic structures are relatively straightforward since they usually rely on the use of periodic boundary conditions. Problems arise, however, when attempting to use these methods to study the thermochemical properties of random solid solutions since an approximation must be made in order to simulate a random atomic configuration through a periodic structure. The usual approaches that have been used in the past can be summarized as follows.

(i) The most direct approach is the supercell method. In this case, the sites of the supercell can be randomly occupied by either $A$ or $B$ atoms to yield the desired $A_{1-x} B_{x}$ composition. In order to reproduce the statistics corresponding to a random alloy, such supercells must necessarily be very large. This approach is, therefore, computationally prohibitive when the size of the supercell is on the order of hundreds of atoms.

(ii) Another technique, the coherent potential approximation $^{6}(\mathrm{CPA})$ method, is a single-site approximation that models the random alloy as an ordered lattice of effective atoms. These are constructed from the criterion that the average scattering of electrons off the alloy components should vanish. ${ }^{7}$ In this method, local relaxations are not considered explicitly and the effects of alloying on the distribution of local environments cannot be taken into account. Local relaxations have been shown to significantly affect the properties of random solutions, ${ }^{8}$ especially when the constituent atoms vary greatly in size and, therefore, their omission constitutes a major drawback. Although the local relaxation energy can be taken into account, ${ }^{7}$ these corrections rely on cluster expansions of the relaxation energy of ordered structures and the distribution of local environments is not explicitly considered. Additionally, such corrections are system specific.

(iii) A third option is to apply the cluster expansion approach. ${ }^{9}$ In this case, a generalized Ising model is used and the spin variables can be related to the occupation of either atom $A$ or $B$ in the parent lattice. In order to obtain an expression for the configurational energy of the solid phase, the energies of multiple configurations (typically in the order of a few dozens) based on the parent lattice must be calculated to obtain the parameters that describe the energy of any given $A_{1-x} B_{x}$ composition. This approach typically relies on 
the calculation of the energies of a few dozen ordered structures.

In the techniques outlined above, there are serious limitations in terms of either the computing power required (supercells, cluster expansion) or the ability to accurately represent the local environments of random solutions (CPA). Ideally, one would like to be able to accurately calculate the thermodynamic and physical properties of a random solution with as small a supercell as possible so that accurate firstprinciples methods can be applied. This has become possible thanks to the development of special quasirandom structures (SQS's).

The concept of SQS was first developed by Zunger et al. ${ }^{10}$ to mimic random solutions without generating a large supercell or using many configurations. The basic idea consists of creating a small-4-48 atoms-periodic structure with the target composition that best satisfies the pair and multisite correlation functions corresponding to a random alloy, up to a certain coordination shell. Upon relaxation, the atoms in the structure are displaced away from their equilibrium positions, creating a distribution of local environments that can be considered to be representative of a random solution, at least up to the first few coordination shells.

Provided the interatomic electronic interactions in a given system are relatively short range, the first-principles calculations of the properties of these designed supercells can be expected to yield sensible results, especially when calculating properties that are mostly dependent on the local atomic arrangements, such as enthalpy of mixing, charge transfer, local relaxations, and so forth. It is important to stress that the approach fails whenever a property depends on longrange interactions.

The SQS's for fcc-based alloys and bcc alloys have been generated by Wei et al. ${ }^{11}$ and Jiang et al. ${ }^{12}$ respectively. However, to the best knowledge of these authors, there has been no investigation on the application of the SQS approach to the study of hep substitutional random solutions. In the present work, we propose two SQS's capable of mimicking hcp random alloys at 25,50 , and 75 at. \%. The paper is organized as follows.

The proposed SQS's are characterized in terms of their ability to reproduce the pair and multisite correlation functions of a truly random hcp solution. Subsequently, the structures are tested in terms of their ability to reproduce, via first-principles calculations, the properties of certain selected stable or metastable binary hcp solutions, namely, Cd-Mg, $\mathrm{Mg}-\mathrm{Zr}$, Al-Mg, Mo-Ru, Hf-Ti, Hf-Zr, and Ti-Zr. To further analyze the relaxation behavior of the structures, the distribution of first nearest bond lengths as well as the radial distribution for the first few coordination shells is presented. Finally, for each of the selected binaries, the calculated and available experimental lattice parameters and enthalpy of mixing are compared. Results from other techniques are also presented where available in order to further corroborate the present calculations.

\section{GENERATION OF SPECIAL QUASIRANDOM STRUCTURES}

In order to characterize the statistics of a given atomic arrangement, one can use its correlation function. ${ }^{13}$ Within the context of lattice algebra, we can assign a "spin value," $\sigma= \pm 1$, to each of the sites of the configuration, depending on whether the site is occupied by $A$ - or $B$-type atoms. Furthermore, all the sites can be grouped in figures, $f(k, m)$, of $k$ vertices, where $k=1,2,3, \ldots$, responds to a shape, point, pair, and triplet,..., respectively, spanning a maximum distance of $m$, where $m=1,2,3, \ldots$, is the first, second, and third-nearest neighbors, and so forth. The correlation functions $\bar{\Pi}_{k, m}$ are the averages of the products of site occupations $( \pm 1$ for binary alloys and $\pm 1,0$ for ternary alloys) of figure $k$ at a distance $m$ and are useful in describing the atomic distribution. The optimum SQS for a given composition is the one that best satisfies the condition

$$
\left(\bar{\Pi}_{k, m}\right)_{\mathrm{SQS}} \cong\left\langle\bar{\Pi}_{k, m}\right\rangle_{R},
$$

where $\left\langle\bar{\Pi}_{k, m}\right\rangle_{R}$ is the correlation function of a random alloy, which is simply by $(2 x-1)^{k}$ in the $A_{1-x} B_{x}$ substitutional binary alloy, where $x$ is the composition. We considered SQS's of two different compositions, i.e., $x=0.5$ and 0.75 .

Unlike cubic structures, the order of a given configuration in the hcp lattices relative to a given lattice site may be altered with the variation of $c / a$ ratio. However, these new arrangements will not cause any change in the correlation functions, since one can thus use any $c / a$ ratio to generate the hcp SQS's. As a matter of simplicity, the ideal $c / a$ ratio was considered in order to generate SQS's.

In the present work, we used the alloy theoretic automation toolkit $(\mathrm{ATAT})^{3}$ to generate special quasirandom structures for the hcp structure of 8 and 16 sites. The schematic diagrams of the created special quasirandom structure with 16 atoms are shown in Fig. 1 and the corresponding lattice vectors and atomic positions are listed in Table I.

The correlation functions of the generated 8- and 16-atom SQS's were investigated to verify that they satisfied at least the short-range statistics of an hep random solution. As is shown in Table II, the 16-atom structures satisfy the pair correlation functions of random alloys up to the fifth and third nearest neighbor for the 50 at. \% and the 75 at. \% compositions, respectively. On the other hand, Table II shows that the SQS-8 for 75 at. \% could not satisfy the random correlation function even for the first-nearest-neighbor pair. Thus, SQS's with 16 atoms are capable of mimicking a random hep configuration beyond the first coordination shell.

It is important to note that in Table II, and contrary to what is observed in the SQS for cubic structures, some figures have more than one crystallographically inequivalent figure at the same distance. For example, in the case of hcp lattices with the ideal $c / a$ ratio, two pairs may have the same interatomic distance and yet be crystallographically inequivalent. In this case, despite the fact that the two pairs $(0,0,0)$ and $(a, 0,0) ;(0,0,0)$ and $\left(\frac{1}{3}, \frac{2}{3}, \frac{1}{2}\right)$, have the same interatomic distance $a$, they do not share the same symmetry operations. This degeneracy is broken when the $c / a$ ratio deviates from its ideal value.

For the sake of efficiency, the initial lattice parameters of the SQS's were determined from Vegard's law. By doing so, the $c / a$ ratio was no longer ideal. Afterwards, we checked the correlation functions of the new structures and found that 


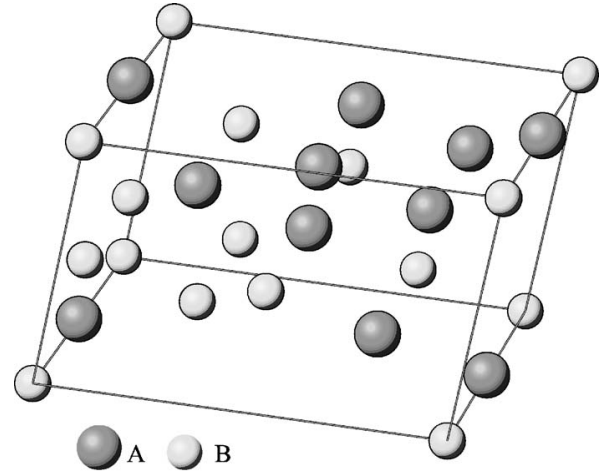

(a)

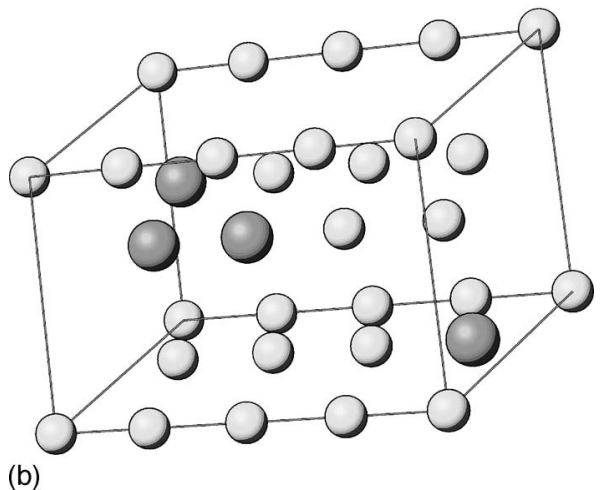

FIG. 1. Crystal structures of the $A_{1-x} B_{x}$ binary hep SQS-16 structures in their ideal, unrelaxed forms. All the atoms are at the ideal hcp sites, even though both structures have the space group $P 1$. (a) SQS-16 for $x=0.5$. (b) SQS-16 for $x=0.75$.

they remained the same as long as the corresponding figures were indentical.

The maximum range over which the correlation function of an SQS mimics that of a random alloy can be increased by increasing the supercell size. As the size of the SQS increases, the probability of finding configurations that mimic random alloys over a wider coordination range increases accordingly. The search algorithm used in this work consists of enumerating every possible supercell of a given volume and for each supercell, enumerating every possible atomic configuration. For each configuration, the correlation functions of different figures, i.e., points, pairs, and triplets, are calculated. To save time, the calculation of the correlations is stopped as soon as one of them does not match the random state value. This algorithm becomes prohibitively expensive very rapidly. The generation of a larger SQS could be accomplished by using a Monte Carlo-like scheme (e.g., Abrikosov et $a l .{ }^{14}$ ), but this is beyond the scope of present work. In fact, the authors could generate a 32-atom SQS's, and the average total energy difference between 16-atom SQS's and 32-atom SQS's in the Cd-Mg system was around $2 \mathrm{meV}$ per atom. The authors maintain a focus on 16-atom SQS, because this size represents a good compromise between accuracy and the computational requirements associated with the necessary first-principles calculations.

It is also important to note that finding a good hep SQS is more difficult than finding an SQS of cubic structures with the same range of matching correlations due to the fact that,
TABLE I. Structural descriptions of the SQS- $N$ structures for the binary hep solid solution. Lattice vectors and atomic positions are given in fractional coordinates of hcp lattice. Atomic positions are given for the ideal, unrelaxed hcp sites.

\begin{tabular}{|c|c|c|c|c|c|c|c|c|}
\hline & \multicolumn{4}{|c|}{$x=0.5$} & \multicolumn{4}{|c|}{$x=0.75$} \\
\hline & \multicolumn{4}{|c|}{ Lattice vectors } & \multicolumn{4}{|c|}{ Lattice vector } \\
\hline & \multirow{3}{*}{\multicolumn{3}{|c|}{$\left(\begin{array}{ccc}0 & -1 & -1 \\
-2 & -2 & 0 \\
-2 & 1 & -1\end{array}\right)$}} & & \multirow{3}{*}{\multicolumn{4}{|c|}{$\left(\begin{array}{ccc}1 & 1 & 1 \\
-1 & 0 & 1 \\
0 & -4 & 0\end{array}\right)$}} \\
\hline & & & & & & & & \\
\hline & & & & & & & & \\
\hline & \multicolumn{4}{|c|}{ Atomic positions } & \multicolumn{4}{|c|}{ Atomic positions } \\
\hline & $-2 \frac{1}{3}$ & $-1 \frac{2}{3}$ & $-1 \frac{1}{2}$ & $A$ & $-\frac{1}{3}$ & $-2 \frac{2}{3}$ & $1 \frac{1}{2}$ & $A$ \\
\hline & -1 & -1 & -1 & $A$ & $-\frac{1}{3}$ & $-1 \frac{2}{3}$ & $1 \frac{1}{2}$ & $A$ \\
\hline & -2 & 0 & -1 & $A$ & 0 & -3 & 2 & $A$ \\
\hline & $-1 \frac{1}{3}$ & $\frac{2}{3}$ & $-1 \frac{1}{2}$ & $A$ & 0 & -3 & 1 & $A$ \\
\hline & -3 & -2 & -1 & $A$ & 0 & -2 & 2 & $B$ \\
\hline \multirow[t]{11}{*}{ SQS-16 } & $-2 \frac{1}{3}$ & $\frac{2}{3}$ & $-1 \frac{1}{2}$ & $A$ & 0 & -1 & 2 & $B$ \\
\hline & -4 & -2 & -2 & $A$ & 0 & 0 & 2 & $B$ \\
\hline & $-3 \frac{1}{3}$ & $-1 \frac{2}{3}$ & $-1 \frac{1}{2}$ & $A$ & $-\frac{1}{3}$ & $-\frac{2}{3}$ & $1 \frac{1}{2}$ & $B$ \\
\hline & -2 & -2 & -1 & $B$ & $-\frac{1}{3}$ & $\frac{1}{3}$ & $1 \frac{1}{2}$ & $B$ \\
\hline & $-1 \frac{1}{3}$ & $-1 \frac{2}{3}$ & $\frac{1}{2}$ & $B$ & $-\frac{1}{3}$ & $-3 \frac{2}{3}$ & $\frac{1}{2}$ & $B$ \\
\hline & -3 & -1 & -1 & $B$ & 0 & -2 & 1 & $B$ \\
\hline & -2 & -1 & -1 & $B$ & $-\frac{1}{3}$ & $-2 \frac{2}{3}$ & $\frac{1}{2}$ & $B$ \\
\hline & $-1 \frac{1}{3}$ & $\frac{2}{3}$ & $\frac{1}{2}$ & $B$ & 0 & -1 & 1 & $B$ \\
\hline & $\frac{1}{3}^{3}$ & $\frac{2}{3}$ & $\frac{1}{2}$ & $B$ & $-\frac{1}{3}$ & $-1 \frac{2}{3}$ & $\frac{1}{2}$ & $B$ \\
\hline & $-2 \frac{1}{3}$ & $-1 \frac{2}{3}$ & $\frac{1}{2}$ & $B$ & 0 & 0 & 1 & $B$ \\
\hline & -3 & -1 & -2 & $B$ & $-\frac{1}{3}$ & $-\frac{2}{3}$ & $\frac{1}{2}$ & $B$ \\
\hline
\end{tabular}

Lattice vectors $\left(\begin{array}{ccc}-1 & 1 & 1 \\ 1 & -1 & 1 \\ 1 & 1 & 0\end{array}\right)$
Lattice vectors

$$
\left(\begin{array}{ccc}
1 & 1 & -1 \\
0 & -1 & -1 \\
-2 & 2 & 0
\end{array}\right)
$$

Atomic position for a given range of correlations, there are more symmetrically distinct correlations to match. Additionally, the lower symmetry of the hcp structure implies that there are also many more candidate configurations to search through in order to find a satisfactory SQS. Thus, the number of distinct supercells is larger and the number of symmetrically distinct atomic configurations is larger, in comparison to fcc or bcc lattices. 
TABLE II. Pair and multisite correlation functions of SQS- $N$ structures when the $c / a$ ratio is ideal. The number in the square bracket next to $\bar{\Pi}_{k, m}$ is the number of equivalent figures at the same distance in the structure, the so-called degeneracy factor.

\begin{tabular}{|c|c|c|c|c|c|c|}
\hline & Random & $\begin{array}{c}x=0.5 \\
\text { SQS-16 }\end{array}$ & SQS-8 & Random & $\begin{array}{l}x=0.75 \\
\text { SQS-16 }\end{array}$ & SQS-8 \\
\hline $\bar{\Pi}_{2,1}[6]$ & 0 & 0 & 0 & 0.25 & 0.25 & 0.16667 \\
\hline $\bar{\Pi}_{2,1}[6]$ & 0 & 0 & 0 & 0.25 & 0.25 & 0.33333 \\
\hline $\bar{\Pi}_{2,2}[6]$ & 0 & 0 & 0 & 0.25 & 0.25 & 0.33333 \\
\hline $\bar{\Pi}_{2,3}[2]$ & 0 & 0 & 0 & 0.25 & 0.25 & 0 \\
\hline $\bar{\Pi}_{2,4}[12]$ & 0 & 0 & 0 & 0.25 & 0.25 & 0.16667 \\
\hline $\bar{\Pi}_{2,4}[6]$ & 0 & 0 & -0.33333 & 0.25 & 0.45833 & 0 \\
\hline $\bar{\Pi}_{2,5}[12]$ & 0 & 0 & -0.33333 & 0.25 & 0.33333 & 0.33333 \\
\hline $\bar{\Pi}_{2,6}[6]$ & 0 & -0.33333 & 0.33333 & 0.25 & 0.16667 & 0.33333 \\
\hline $\bar{\Pi}_{2,7}[12]$ & 0 & 0 & 0 & 0.25 & 0.25 & 0.5 \\
\hline $\bar{\Pi}_{2,8}[12]$ & 0 & 0 & 0 & 0.25 & 0.1667 & 0.33333 \\
\hline $\bar{\Pi}_{3,1}[12]$ & 0 & 0 & 0.33333 & 0.125 & -0.08333 & 0.16667 \\
\hline $\bar{\Pi}_{3,1}[2]$ & 0 & 0 & 0 & 0.125 & 0.25 & 0.5 \\
\hline $\bar{\Pi}_{3,1}[2]$ & 0 & 0 & 0 & 0.125 & 0.25 & 0.5 \\
\hline $\bar{\Pi}_{3,2}[24]$ & 0 & 0 & 0 & 0.125 & -0.04167 & 0 \\
\hline $\bar{\Pi}_{3,3}[6]$ & 0 & 0 & 0 & 0.125 & -0.08333 & 0.16667 \\
\hline $\bar{\Pi}_{3,3}[6]$ & 0 & 0 & 0 & 0.125 & -0.08333 & -0.16667 \\
\hline $\bar{\Pi}_{4,1}[4]$ & 0 & 0 & 0 & 0.0625 & 0 & 0.5 \\
\hline $\bar{\Pi}_{4,2}[12]$ & 0 & 0 & -0.33333 & 0.0625 & -0.16667 & -0.16667 \\
\hline $\bar{\Pi}_{4,2}[12]$ & 0 & 0 & 0 & 0.0625 & 0 & 0 \\
\hline $\bar{\Pi}_{4,3}[6]$ & 0 & 0.33333 & 0.33333 & 0.0625 & -0.16667 & 0 \\
\hline
\end{tabular}

In order to verify the proposed 16-atom SQS's are adequate for the simulation of hcp random solutions, the authors calculated other SQS's at 75 at. \% which have randomlike pair correlations up to the third nearest neighbor but that have slightly different correlations for the fourth nearest neighbor. The pair correlation function at 75 at. \% of a truly random solution would be $(2 \times 0.75-1)^{2}=0.25$ and therefore the four SQS's in Table III are worse than the one used in the present work. These structures were applied to the $\mathrm{Cd}$ 25 at. $\%-\mathrm{Mg} 75$ at. \% system and, as can be seen in Table III, the associated energy differences are negligible. This is due to the fact that the energetics of this system are dominated by short-range interactions. Thus, as long as the most important pair correlations (up to the third nearest neighbors in hcp structure with ideal $c / a$ ratio) are satisfied, the SQS's can successfully be applied to acquire properties of random solutions in which short-range interactions dominate.

\section{FIRST-PRINCIPLES METHODOLOGY}

The selected hcp SQS-16 structures were used as geometrical input for the first-principles calculations. The Vienna $A b$ initio Simulation Package ${ }^{15}$ (VASP) was used to perform the density functional theory electronic structure calculations. The projector augmented wave method ${ }^{16}$ was chosen and the general gradient approximation ${ }^{17}$ was used to take into account exchange and correlation contributions to

TABLE III. Pair correlation functions up to the fifth and the calculated total energies of other 16 atoms SQS's for $\mathrm{Cd}_{0.25} \mathrm{Mg}_{0.75}$ are enumerated to be compared with the one used in this work (SQS-16). The total energies are given in unit's of eV/atom.

\begin{tabular}{lccccc}
\hline \hline & $a$ & $b$ & $c$ & $d$ & SQS-16 \\
\hline $\bar{\Pi}_{2,1}[6]$ & 0.25 & 0.25 & 0.25 & 0.25 & 0.25 \\
$\bar{\Pi}_{2,1}[6]$ & 0.25 & 0.25 & 0.25 & 0.25 & 0.25 \\
$\bar{\Pi}_{2,2}[6]$ & 0.25 & 0.25 & 0.25 & 0.25 & 0.25 \\
$\bar{\Pi}_{2,3}[2]$ & 0.25 & 0.25 & 0.25 & 0.25 & 0.25 \\
$\bar{\Pi}_{2,4}[12]$ & 0.20833 & 0.16667 & 0.16667 & 0.08333 & 0.25 \\
$\bar{\Pi}_{2,4}[6]$ & 0.5 & 0.5 & 0.5 & 0.16667 & 0.45833 \\
$\bar{\Pi}_{2,5}[12]$ & 0.5 & 0.16667 & 0.33333 & 0.33333 & 0.33333 \\
Symmetry & -1.3864 & -1.3882 & -1.3886 & -1.3886 & -1.3869 \\
preserved & & & & & \\
Fully & -1.3874 & -1.3887 & -1.3889 & -1.3893 & -1.3883 \\
relaxed & & & & & \\
\hline \hline
\end{tabular}


the Hamiltonian of the ion-electron system. A constant energy cutoff of $350 \mathrm{eV}$ was used for all the structures, with $5000 k$ points per reciprocal atom based on the MonkhorstPack scheme for the Brillouin-zone integrations. The $k$-point meshes were centered at the $\Gamma$ point. The convergence criterion for the calculations was $10 \mathrm{meV}$ with respect to the 16 atoms. Spin-polarization was not taken into account. The generated SQS's were either fully relaxed, or relaxed without allowing local ion relaxations, i.e., only volume and $c / a$ ratio were optimized. As will be seen below, the full relaxation caused some of the SQS's to lose the original hcp symmetry.

\section{RESULTS AND DISCUSSIONS}

\section{A. Analysis of relaxed structures}

The symmetry of the resulting SQS was checked using the PLATON code $^{18}$ before and after the relaxations. Both SQS's have the lowest symmetry of P1, although all the atoms are sitting on the lattice sites of hcp. The procedure was verified by checking the symmetries of the generated unrelaxed SQS. Once all the sites in the SQS were substituted with one single atomic species, PLATON identified SQS's as perfect hcp structures. All the atoms of the initial structures are on their exact hcp lattice sites. However, upon relaxation the atoms may be displaced from these ideal positions. According to the definition of an hcp random solution, all the atoms, in this case two different type of atoms, should be at the hcp lattice points-within a certain tolerance-even after the structure has been fully relaxed. The default tolerance of detecting the symmetry of the relaxed structures allowed the atoms to deviate from their original lattice sites by up to $20 \%$.

In principle, relaxations should be performed with respect to the degrees of freedom consistent with the initial symmetry of any given configuration. In the particular case of the hcp SQS's, local relaxations may in some cases be so large that the character of the underlying parent lattice is lost. However, within the CALPHAD methodology, one has to define the Gibbs energy of a phase throughout the entire composition range, regardless of whether the structure is stable or not. In these cases, it is necessary to constrain the relaxations so that they are consistent with the lattice vectors and atom positions of an hcp lattice. Obviously, the energetic contributions due to local relaxations are not considered in this case. The results of these constrained relaxations can therefore be directly compared to those calculations using the CPA. In most cases, local relaxations were not significant. However, in a few instances, it was found that the structure was too distorted to be considered as hcp after the full relaxation. However, this symmetry check was not sufficient to characterize the relaxation behavior of the relaxed SQS. Furthermore, in some of the cases it may be possible for the structure to fail the symmetry test and still retain an hcp-like environment within the first couple of coordination shells, implying that the energetics and other properties calculated from these structures could be characterized as reasonable, although not optimal, approximations of random configurations.

\section{Radial distribution analysis}

In order to investigate the local relaxation of the fully relaxed SQS, their radial distribution (RD) was analyzed. Through this analysis, the bond distribution and coordination shells were studied to determine whether the relaxed structures maintained the local hcp-like environment they were supposed to mimic in the first place. Additionally, this analysis permitted us to quantify the degree of local relaxations up to the fifth coordination shells.

The RD of each of the fully relaxed structures was obtained by counting the number of atoms within bins of $10^{-3} \AA$, up to the fifth coordination shell. In order to eliminate high frequency noise, the raw data was scaled and smoothed through Gaussian smearing with a characteristic distance of $0.01 \AA$. Pseudo-Voigt functions were then used to fit each of the smoothed peaks and the goodness of fit was in part determined through the summation of the total areas of the peaks and comparing them to the total number of atoms that were expected within the analyzed coordination shells. The relaxation of the atoms at each coordination shell is quantified by the width of the corresponding peak in the fitted RD.

The RD results of selected SQS's are given in Fig. 2. The unrelaxed, fully relaxed, and nonlocally relaxed structures are compared in each case as well as the smoothed bond distributions and their fitted curves. These results are representative of the RD's obtained for the seven binary systems at the three compositions studied.

Figure 2(a) shows the RDs for the Hf-Zr SQS at the 50 at. \% composition. As can be seen in the figure, the RDs for the unrelaxed and nonlocally relaxed SQS are almost identical, implying that in this system Vegard's Law is closely followed. Furthermore, the RD for the fully relaxed SQS in Fig. 2(b) shows a rather narrow distribution around each of the the bondlengths corresponding to the ideal or unrelaxed structure. The system therefore needs to undergo very negligible local relaxations in order to minimize its energy.

In the case of the Cd-Mg solution at 50 at. \% [Fig. 2(c)], the RDs of the unrelaxed and nonlocally relaxed SQS are more dissimilar. Even in the nonlocally relaxed calculation, the original first coordination shell (corresponding to the six first-nearest neighbors) has split into two different shells (of 4 and 2 atoms) and the position of the peak is noticeably shifted. The first two well defined coordination shells of the unrelaxed structure have merged into a single, broad peak at $3.14 \AA$ upon full relaxation, as shown in Fig. 2(d). This peak now encloses 12 first nearest neighbors. As shown in Table II, $\bar{\Pi}_{2,1}$ and $\bar{\Pi}_{2,4}$ have two differnet types of pairs. However, since they have the same correlation functions, they cannot be distinguished. In Fig. 2(d) it is also shown how the fourth and fifth coordination shells merge at $5.40 \AA$, enclosing 18 atoms. It can be expected that if the $c / a$ ratio of a relaxaed structure is close to ideal and the broadening of nearby shells are wide enough that they merge, then the structure has almost the same radial distribution of an ideal hcp structure, albeit with a large peak width.

Figure 2(e) shows the RD for the $\mathrm{Mg}_{50} \mathrm{Zr}_{50}$ composition. Among the three RD's presented in Fig. 2, this one is clearly 

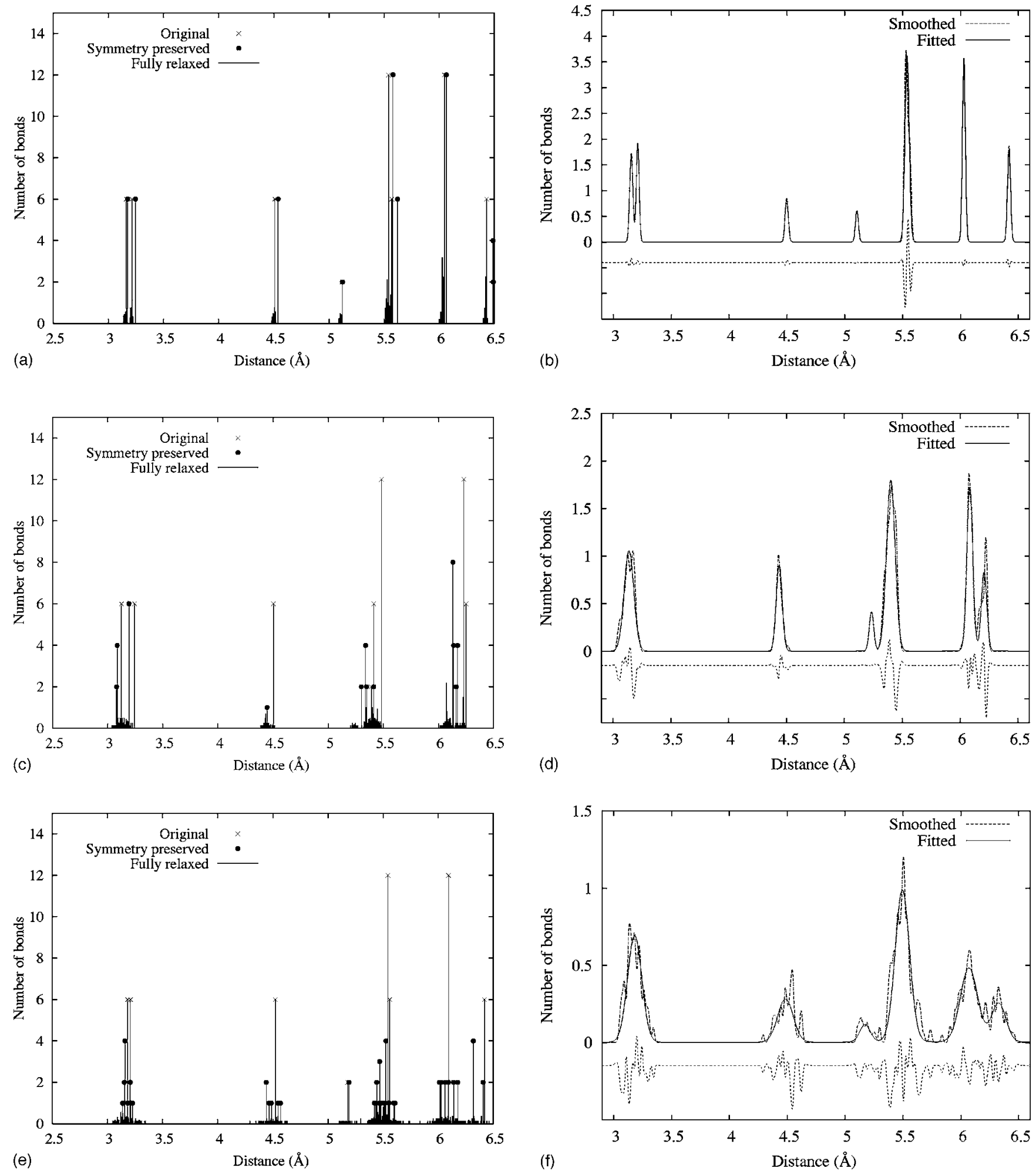

FIG. 2. Radial distribution analysis of selected SQS's. The dotted lines under the smoothed and fitted curves are the error between the two curves. (a) $\mathrm{RD}$ of $\mathrm{Hf}_{50} \mathrm{Zr}_{50}\left(\Delta H_{\text {mix }} \sim 0\right.$ ). (b) Smoothed and fitted RD's of fully relaxed $\mathrm{Hf}_{50} \mathrm{Zr}_{50}$. (c) $\mathrm{RD}$ of $\mathrm{Cd}_{50} \mathrm{Mg}_{50}\left(\Delta H_{\text {mix }}<0\right.$ ). (d) Smoothed and fitted RD's of fully relaxed $\mathrm{Cd}_{50} \mathrm{Mg}_{50}$. (e) $\mathrm{RD}$ of $\mathrm{Mg}_{50} \mathrm{Zr}_{50}\left(\Delta H_{\text {mix }}>0\right)$. (f) Smoothed and fitted RD's of fully relaxed $\mathrm{Mg}_{50} \mathrm{Zr}_{50}$.

the one that undergoes the greatest distortion upon full relaxation. Even in the nonlocally relaxed structures there is a broad bondlength distribution around the peaks of the unrelaxed SQS. With respect to the fully relaxed SQS, it can be seen how the peaks for the fifth and sixth coordination shells have practically merged. In this case, the local environment of each atom within the SQS stops being hcp-like within the first couple of coordination shells. Although the two end 
TABLE IV. Results of radial distribution analysis for the seven binaries studied in this work. FWHM shows the averaged full width at half maximum and is given in $\AA$. Errors indicate the difference in the number of atoms calculated through the sum of peak areas and those expected in each coordination shell.

\begin{tabular}{lcccccccc}
\hline \hline Compositions & & Cd-Mg & Mg-Zr & Al-Mg & Mo-Ru & Hf-Ti & Hf-Zr & Ti-Zr \\
\hline & FAHM & $0.06 \pm 0.01$ & $0.09 \pm 0.03$ & $0.08 \pm 0.02$ & N/A & $0.11 \pm 0.03$ & $0.02 \pm 0.00$ & $0.16 \pm 0.05$ \\
$\mathrm{~A}_{75} \mathrm{~B}_{25}$ & Error, $\%$ & 0.72 & 0.39 & 0.47 & N/A & 1.07 & 1.84 & 1.27 \\
& Symmetry & PASS & PASS & PASS & FAIL & PASS & PASS & FAIL \\
& FWHM & $0.07 \pm 0.02$ & $0.15 \pm 0.02$ & $0.15 \pm 0.07$ & $0.13 \pm 0.01$ & $0.16 \pm 0.02$ & $0.03 \pm 0.01$ & $0.09 \pm 0.06$ \\
$\mathrm{~A}_{50} \mathrm{~B}_{50}$ & Error, $\%$ & 0.30 & 1.42 & 1.28 & 1.90 & 0.35 & 1.84 & 2.39 \\
& Symmetry & PASS & FAIL & FAIL & PASS & PASS & PASS & PASS \\
& FWHM & $0.04 \pm 0.01$ & $0.09 \pm 0.03$ & $0.10 \pm 0.02$ & $0.07 \pm 0.02$ & $0.11 \pm 0.06$ & $0.03 \pm 0.00$ & $0.13 \pm 0.07$ \\
$\mathrm{~A}_{25} \mathrm{~B}_{75}$ & Error, $\%$ & 2.05 & 1.22 & 0.26 & 1.93 & 0.26 & 1.01 & 0.96 \\
& Symmetry & PASS & PASS & PASS & PASS & PASS & PASS & PASS \\
\hline \hline
\end{tabular}

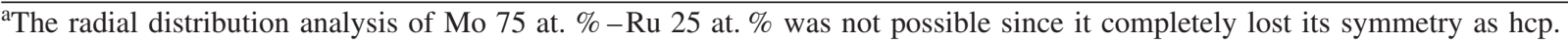

members of this binary alloy have an hcp as the stable structure, it is evident from this figure that the SQS arrangement is unstable and there is a tendency for the structure to distort. In this system, there is a miscibility gap in the hcp phase up to $\sim 900 \mathrm{~K}$ and the RD reflects the tendency for the system to phase separate.

The results from the peak fitting for all the fully relaxed SQS's are summarized in Table IV. It should be noted that regardless of the system and compositions, the sum of the areas under each peak should converge to a single value, proportional to 50 atoms. For each peak, the error was quantified as the absolute and normalized difference between the expected and actual areas. The error reported in the table is the averaged value for all the peaks in the RD. The broadness of the peaks in the RD is quantified through the full width at half maximum (FWHM). In the table, the reported FWHM corresponds to the average FWHM observed for the coordination shells enclosing a total of 50 atoms. Note that the alloys with the smallest FWHM are $\mathrm{Hf}-\mathrm{Zr}$ and $\mathrm{Cd}-\mathrm{Mg}$. As will be seen later, $\mathrm{Hf}-\mathrm{Zr}$ behaves almost ideally and $\mathrm{Cd}-\mathrm{Mg}$ is a system with rather strong attractive interactions between unlike atoms that forms ordered hexagonal structures at the 25 and 75 at. \% compositions.

\section{Bond length analysis}

In addition to the $\mathrm{RD}$ analysis, we performed the bondlength analysis $(A-A, B-B$, and $A-B)$ for all the relaxed SQS's. In Table $\mathrm{V}$ the bond lengths corresponding to the first nearest neighbors for all the 21 SQS's are presented. As expected, in the majority of the cases the sequence $d_{i i}<d_{i j}$ $<d_{j j}$ is observed throughout the composition range, where $d_{i j}$ corresponds to the bond distance between two different atom types. The two notable exceptions to this trend correspond to the $\mathrm{Cd}-\mathrm{Mg}$ and $\mathrm{Mg}-\mathrm{Zr}$ alloys. As will be mentioned below, the $\mathrm{Cd}-\mathrm{Mg}$ system tends to form rather stable intermetallic compounds at the 25, 50, and 75 at. \% compositions, including two hexagonal intermetallic compounds. The calculated enthalpy of mixing in this case-shown in Fig. 3(a) - is the most negative among seven binaries studied and the fact that the Cd-Mg bonds are shorter than $\mathrm{Cd}-\mathrm{Cd}$ and

TABLE V. First nearest-neighbor average bondlengths for the fully relaxed hcp SQS of the seven binaries studied in this work. Uncertainty corresponds to the standard deviation of the bondlength distributions.

\begin{tabular}{ccccccccc}
\hline \hline Compositions & Bonds & Cd-Mg & Mg-Zr & Al-Mg & Mo-Ru & Hf-Ti & Hf-Zr & Ti-Zr \\
\hline$A_{100} B_{0}$ & $A-A$ & 3.07 & 3.18 & 2.87 & 2.75 & 3.13 & 3.13 & 2.87 \\
& $A-A$ & $3.17 \pm 0.10$ & $3.18 \pm 0.03$ & $2.92 \pm 0.03$ & & $3.14 \pm 0.05$ & $3.18 \pm 0.03$ & $2.96 \pm 0.07$ \\
$A_{75} B_{25}$ & $A-B$ & $3.16 \pm 0.11$ & $3.18 \pm 0.05$ & $2.95 \pm 0.03$ & N/A & $3.10 \pm 0.05$ & $3.18 \pm 0.03$ & $3.02 \pm 0.07$ \\
& $B-B$ & $3.18 \pm 0.10$ & $3.12 \pm 0.10$ & $2.96 \pm 0.03$ & & $3.09 \pm 0.06$ & $3.18 \pm 0.04$ & $3.04 \pm 0.06$ \\
& $A-A$ & $3.16 \pm 0.04$ & $3.16 \pm 0.04$ & $2.98 \pm 0.06$ & $2.81 \pm 0.08$ & $3.09 \pm 0.06$ & $3.18 \pm 0.03$ & $3.00 \pm 0.09$ \\
$A_{50} B_{50}$ & $A-B$ & $3.12 \pm 0.04$ & $3.20 \pm 0.06$ & $3.02 \pm 0.06$ & $2.75 \pm 0.04$ & $3.05 \pm 0.07$ & $3.19 \pm 0.03$ & $3.06 \pm 0.08$ \\
& $B-B$ & $3.15 \pm 0.03$ & $3.14 \pm 0.08$ & $3.07 \pm 0.08$ & $2.75 \pm 0.04$ & $3.00 \pm 0.06$ & $3.20 \pm 0.03$ & $3.12 \pm 0.08$ \\
& $A-A$ & $3.16 \pm 0.01$ & $3.15 \pm 0.04$ & $3.06 \pm 0.04$ & $2.73 \pm 0.04$ & $3.02 \pm 0.05$ & $3.19 \pm 0.03$ & $3.09 \pm 0.08$ \\
$A_{25} B_{75}$ & $A-B$ & $3.14 \pm 0.02$ & $3.19 \pm 0.04$ & $3.08 \pm 0.04$ & $2.73 \pm 0.04$ & $3.00 \pm 0.06$ & $3.19 \pm 0.03$ & $3.11 \pm 0.06$ \\
& $B-B$ & $3.15 \pm 0.01$ & $3.18 \pm 0.04$ & $3.11 \pm 0.03$ & $2.71 \pm 0.04$ & $2.95 \pm 0.05$ & $3.20 \pm 0.04$ & $3.17 \pm 0.06$ \\
$A_{0} B_{100}$ & $B-B$ & 3.18 & 3.19 & 3.18 & 2.68 & 2.87 & 3.19 & 3.19 \\
\hline \hline
\end{tabular}



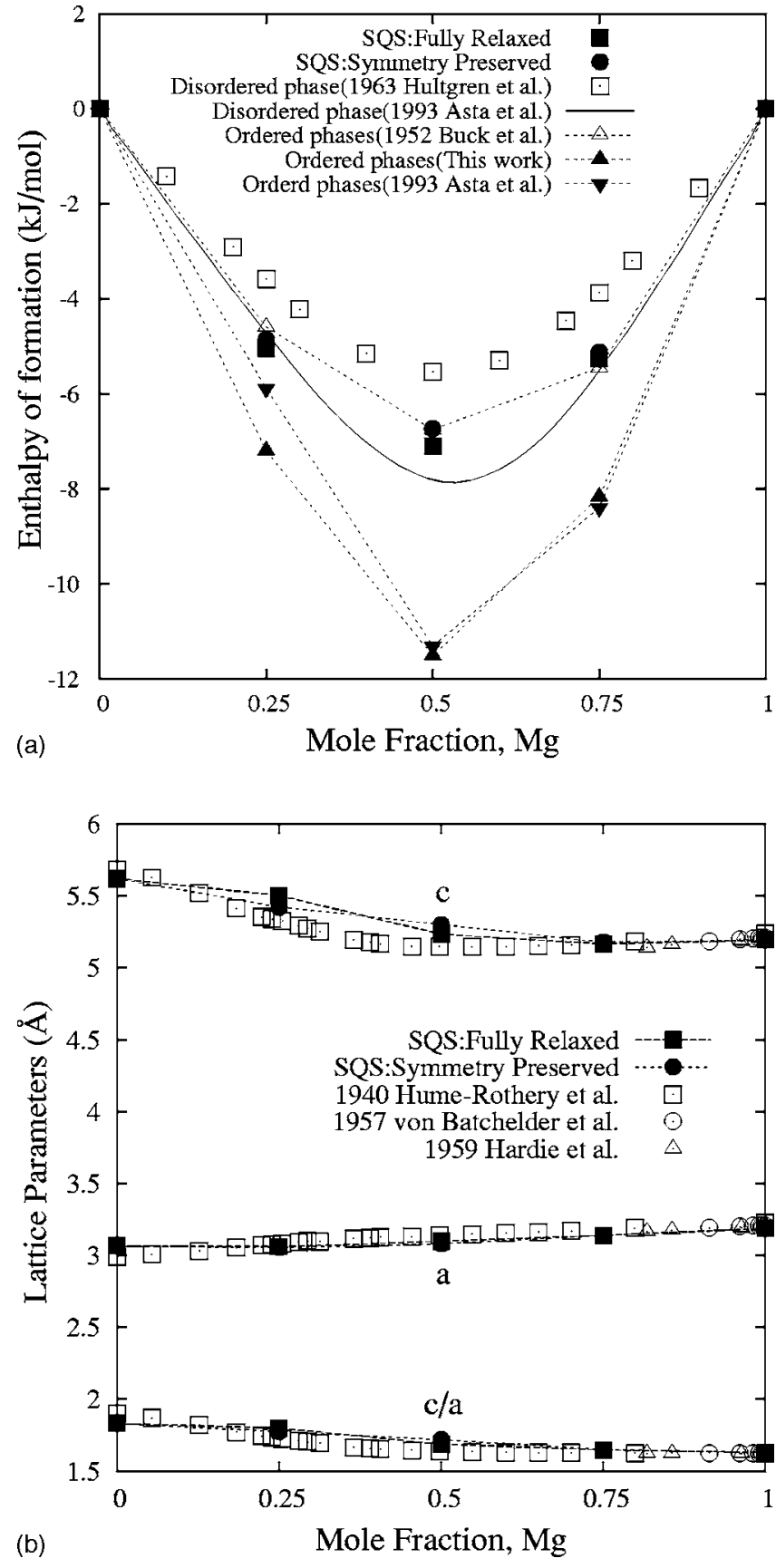

FIG. 3. Calculated and experimental results of mixing enthalpy and lattice parameters for the Cd-Mg system. (a) Calculated enthalpy of mixing for the disordered hcp phase in the Cd-Mg system with SQS at $T=0 \mathrm{~K}$, cluster variation method (CVM) (Ref. 20) at $T=900 \mathrm{~K}$, and experiment (Ref. 21) at $T=543 \mathrm{~K}$. (b) Calculated lattice parameters of the $\mathrm{Cd}-\mathrm{Mg}$ system compared with experimental data (Refs. 22-24).

$\mathrm{Mg}-\mathrm{Mg}$ seems to reflect the tendency of this system to order. In the case of the $\mathrm{Mg}-\mathrm{Zr}$ alloys, the $\mathrm{Mg}-\mathrm{Zr}$ bonds are longer than $\mathrm{Mg}-\mathrm{Mg}$ and $\mathrm{Zr}-\mathrm{Zr}$, suggesting that this system has a great tendency to phase separate, as indicated by the presence of a large hcp miscibility gap in the $\mathrm{Mg}-\mathrm{Zr}$ phase diagram. ${ }^{19}$

\section{B. Enthalpy of mixing}

It is obvious that if an hcp SQS alloy is not stable with respect to local relaxations, its properties are not accessible through experimental measurements. However, approximate effective properties could still be estimated through CALPHAD modeling. In order to compare the energetics and properties of the calculated SQS's with the available experiments or previous thermodynamic models, only the nonlocally relaxed structures were considered whenever the SQS was identified as unstable. This effectively assumes that the structures in question are constrained to maintain their symmetry. The total energies of the structures under symmetry-preserving relaxations are obviously higher since the relaxation energy is not considered. However, we can consider these calculated thermochemical properties as an upper bound which can still be of great use when attempting to generate thermodynamically consistent models based on the combined first-principles/CALPHAD approach.

As mentioned earlier, obtaining thermodynamic properties of random alloys using cluster expansion or the CPA method has some drawbacks. These methods, however, have the advantage of calculating the properties of random alloys at arbitrary and closely spaced concentrations. SQS's in this case are at a disadvantage since the size of the SQS itself limits the concentrations with randomlike correlations. Nevertheless, if we can acquire the properties at these three compositions, we can sufficiently describe the tendency of the system. Furthermore, these SQS's can be applied directly to other binary systems without any modifications.

The enthalpies of mixing for these alloys were calculated at the 25, 50, and 75 at. $\%$ concentrations through the expression

$$
\Delta H\left(A_{1-x} B_{x}\right)=E\left(A_{1-x} B_{x}\right)-(1-x) E(A)-x E(B),
$$

where $E(A)$ and $E(B)$ are the reference energies of the pure components in their hcp ground state.

In the following sections, the generated SQS's are tested by calculating the crystallographic, thermodynamic, and electronic properties of hcp random solutions in seven binary systems Cd-Mg, Mg-Zr, Al-Mg, Mo-Ru, Hf-Ti, Hf-Zr, and Ti-Zr. The results of the calculations are then compared with existing experimental information as well as previous calculations.

\section{C. $\mathrm{Cd}-\mathrm{Mg}$}

In the Cd-Mg system, both elements have the same valence and almost the same atomic volumes. Consequently, there is a wide hcp solid solution range as well as order/ disorder transitions in the central, low temperature region of the phase diagram. In fact, at the 25 and 75 at. \% compositions there are ordered intermetallic phases with hexagonal symmetries.

Figure 3(a) compares the enthalpy of mixing calculated from the fully relaxed and symmetry preserved SQS with the results from cluster expansion. ${ }^{20}$ The results by Asta et al. ${ }^{20}$ at $900 \mathrm{~K}$ are presented for comparison since it is to be expected that these values would be rather close to the calculated enthalpy of completely disordered structures. The pre- 
vious and current calculations are also compared with the experimental measurements as reported in Hultgren $^{21}$ at $543 \mathrm{~K}$. The first thing to note from Fig. 3(a) is that the fully relaxed and symmetry preserved calculations are very close in energy, implying negligible local relaxation. Additionally, the present calculations are remarkably close $(\sim 1 \mathrm{~kJ} / \mathrm{mol})$ to the experimental measurements. By comparing the SQS enthalpy of mixing with the results from the cluster expansion calculations,${ }^{20}$ it is obvious that the former is, at least in this case, more capable of reproducing the experimental measurements.

Formation enthalpies of the three ordered phases in the $\mathrm{Cd}-\mathrm{Mg}$ system, $\mathrm{Cd}_{3} \mathrm{Mg}$, CdMg, and $\mathrm{CdMg}_{3}$ are also presented. The measurements from Hultgren ${ }^{21}$ deviate from the calculated results from Asta et al. ${ }^{20}$ and this work. Cd and $\mathrm{Mg}$ are known as very active elements and it is likely that reaction with oxygen present during the measurements may have introduced some systematic errors. Furthermore, the measurements were conducted at relatively low temperatures, making it difficult for the systems to equilibrate. Nevertheless, experiments and calculations agree that these three compounds constitute the ground state of the $\mathrm{Cd}-\mathrm{Mg}$ system.

Figure 3(b) also shows that the present calculations are able to reproduce the available measurements on the variation of the lattice parameters of hcp Cd-Mg alloys with composition, as well as the deviation of these parameters from Vegard's Law. This deviation is mainly related to the rather large difference in $c / a$ ratio between $\mathrm{Cd}$ and $\mathrm{Mg}$. The $c / a$ ratio of $\mathrm{Cd}$ is one of the largest ones of all the stable hcp structures in the periodic table.

\section{Mg-Zr}

The Mg-Zr system is important due to the grain refining effects of $\mathrm{Zr}$ in magnesium alloys. According to the assessment of the available experimental data by Nayeb-Hashemi and Clark, ${ }^{19}$ the Mg-Zr system shows very little solubility in the three solution phases, bcc, hcp, and liquid. In fact, the low temperature hcp phase exhibits a broad miscibility gap up to $923 \mathrm{~K}$, corresponding to the peritectic reaction hcp + liquid $\rightarrow$ hcp. $^{19}$

Our calculations yielded a positive enthalpy of mixing, confirming the trends derived from the thermodynamic model developed by Hämäläinen et al. ${ }^{25}$ In the case of the full relaxation, however, it was observed that the $\mathrm{Mg}_{50} \mathrm{Zr}_{50}$ SQS was unstable with respect to local relaxations. The instability at this composition and the large, positive enthalpy of mixing indicate that the system has a strong tendency to phase separate. By comparing the fully relaxed and the nonlocally relaxed structures, we estimate that the local relaxation energy lowers the mixing enthalpy of the random hcp SQS by about $2 \mathrm{~kJ} / \mathrm{mol}$ in this system.

Figure 4 shows the calculated mixing enthalpy for the $\mathrm{Mg}-\mathrm{Zr}$ hcp SQS with no local relaxations, as well as the mixing enthalpy calculated from the thermodynamic model by Hämäläinen et al. ${ }^{25}$ which was fitted only through phase diagram data. It is therefore remarkable that the maximum difference between the CALPHAD model and the present hcp SQS calculations is $\sim 3 \mathrm{~kJ} / \mathrm{mol}$. The CALPHAD model,

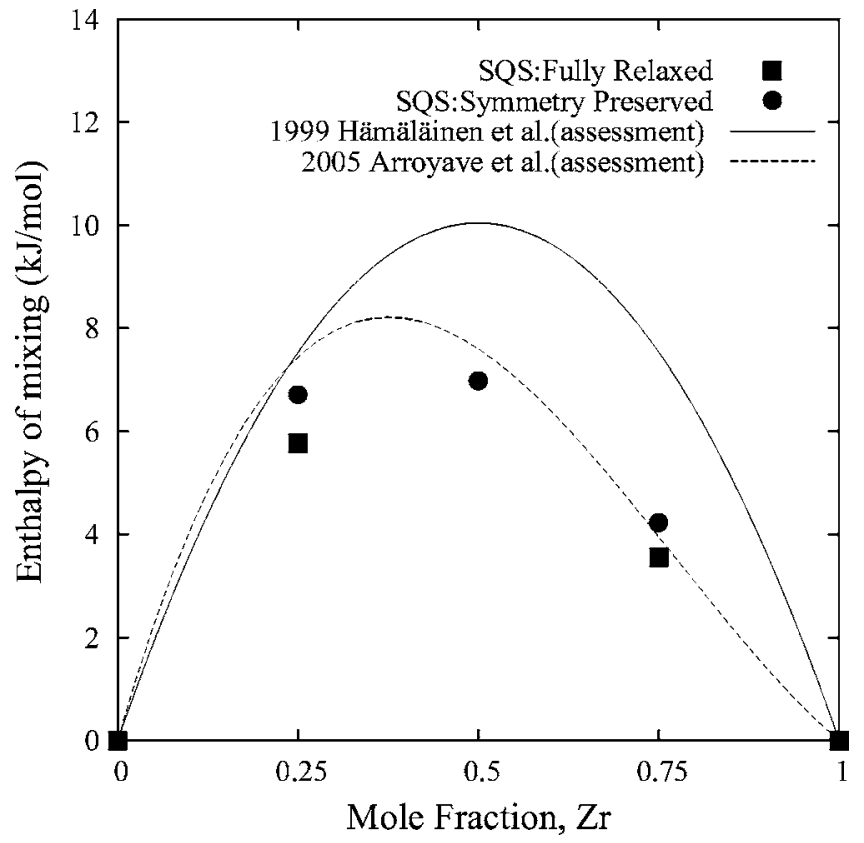

FIG. 4. Calculated enthalpy of mixing in the $\mathrm{Mg}-\mathrm{Zr}$ system compared with a previous thermodynamic assessment (Ref. 25). Both reference states are the hcp structure.

however, does not correctly describe the asymmetry of the mixing enthalpy indicated by the first-principles calculations. The results of the hep SQS calculations for the Mg-Zr system have recently been used to obtain a better thermodynamic description of the $\mathrm{Mg}-\mathrm{Zr}$ system ${ }^{26}$ and, as can be seen in the figure, this description is better at describing the trends in the calculated enthalpy of mixing.

\section{E. Al-Mg}

As one of the most important industrial alloys, the Al-Mg system has been studied extensively recently. ${ }^{27-29}$ This system has two eutectic reactions and shows solubility within both the fcc and hcp phases. However, the solubility ranges are not wide enough so there is only limited experimental information for the properties of the hcp phase. The maximum equilibrium solubility of $\mathrm{Al}$ in the $\mathrm{Mg}$-rich hcp phase is around 12 at. $\%$.

In Fig. 5(a) the calculated enthalpy of mixing is slightly positive. The fully relaxed calculations show that the SQS with the 50 at. \% composition was unstable with respect to local relaxations. This can be explained by the strong interaction between $\mathrm{Al}$ and $\mathrm{Mg}$, as evident from the tendency of this system to form intermetallic compounds at the middle of the phase diagram, such as $\beta-\mathrm{Al}_{140} \mathrm{Mg}_{89}, \gamma-\mathrm{Al}_{12} \mathrm{Mg}_{17}$, and $\varepsilon-\mathrm{Al}_{30} \mathrm{Mg}_{23}$. At the 25 and 75 at. \% compositions the SQS's were stable with respect to local relaxations because both elements have a close-packed structure. Furthermore, at these compositions either the fcc or hcp phase take part in equilibria with some other (intermetallic) phase. Figure 5(a) shows that the present fully relaxed calculations are in excellent agreement with the most recent CALPHAD assessments. ${ }^{27,28}$ Note also that in this case, and contrary to what is observed in the Cd-Mg binary, the energy change 

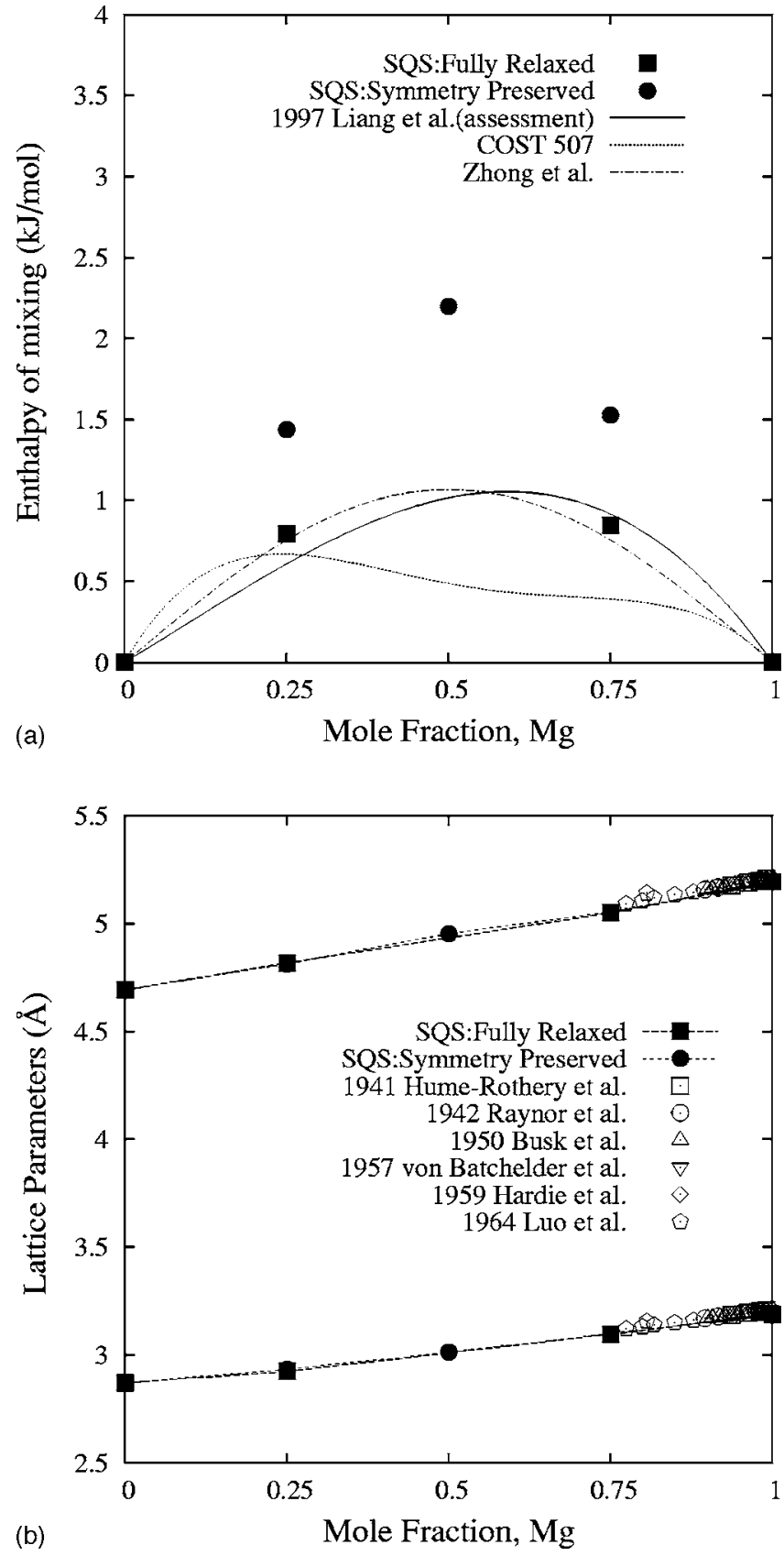

FIG. 5. Calculated and experimental results of mixing enthalpy and lattice parameters for the Al-Mg system. (a) Calculated enthalpy of mixing for the hcp phase in the Al-Mg system compared with assessed data (Ref. 27-29). Reference states are hcp for both elements. (b) Calculated lattice parameters of the hcp phase in the Al-Mg system compared with experimental data (Refs. 23, 24, and 30-33).

associated with local relaxation is not negligible, although it is still within $\sim 1 \mathrm{~kJ} / \mathrm{mol}$.

Additionally, the calculated lattice parameters agree very well with the experimental measurements of Mg-rich hcp alloys, as can be seen in Fig. 5(b). It is important to note that the lattice parameter measurements of metastable hcp alloys from Luo et al. ${ }^{30}$ (77.4 and $87.8 \mathrm{Mg}$ at. \%) are lying on the extrapolated line between the 75 at. \% SQS and the pure $\mathrm{Mg}$ calculations. This is another example of how SQS's can be successfully used in calculating the properties of an hcp solid solution system with narrow solubility range and mixed with non-hcp elements, even in the metastable regions of the phase diagram.

\section{F. Mo-Ru}

The Mo-Ru system shows a wide solubility range within both the bcc and hcp sides of the phase diagram. In the $\mathrm{Ru}$-rich side, the maximum solubility of Mo in the hcp-Ru matrix is up to 50 at. $\%$. The calculations at $\mathrm{Mo}_{25} \mathrm{Ru}_{75}$ and $\mathrm{Mo}_{50} \mathrm{Ru}_{50}$ retained the original hcp symmetry but $\mathrm{Mo}_{75} \mathrm{Ru}_{25}$ did not. The instability of the Mo-rich SQS is not surprising since the Mo-rich bcc region is stable over a wide region of the phase diagram. As shown in Wang et al., ${ }^{34}$ elements whose ground state is bcc are not stable in an hcp lattice and vice versa (bcc Ti, Zr, and $\mathrm{Hf}$ are only stabilized at high temperature due to anharmonic effects). Thus hcp compositions close to the bcc-side would be dynamically unstable and would have a very large driving force to decrease their energy by transforming to bcc.

Recently, Kissavos et al. ${ }^{7}$ calculated the enthalpy of mixing for disordered hcp Mo-Ru alloys through the CPA in which relaxation energies were estimated by locally relaxing selected multisite atomic arrangements. Enthalpy of formation for hcp solutions were calculated from Eq. (3) shown below. The enthalpy of mixing of the disordered hcp phase can be evaluated accordingly based on the so-called lattice stability $^{2} E^{\mathrm{bcc}}(\mathrm{Mo})-E^{\mathrm{hcp}}(\mathrm{Mo})$ :

$$
\begin{aligned}
& \Delta H_{f}\left(\mathrm{Mo}_{1-x} \mathrm{Ru}_{x}\right) \\
&=E^{\mathrm{hcp}}\left(\mathrm{Mo}_{1-x} \mathrm{Ru}_{x}\right)-(1-x) E^{\mathrm{bcc}}(\mathrm{Mo})-x E^{\mathrm{hcp}}(\mathrm{Ru}) \\
&=E^{\mathrm{hcp}}\left(\mathrm{Mo}_{1-x} \mathrm{Ru}_{x}\right)-(1-x) E^{\mathrm{hcp}}(\mathrm{Mo})-x E^{\mathrm{hcp}}(\mathrm{Ru}) \\
&-(1-x) E^{\mathrm{bcc}}(\mathrm{Mo})+(1-x) E^{\mathrm{hcp}}(\mathrm{Mo}) \\
&= H_{\mathrm{mix}}^{\mathrm{hcp}}\left(\mathrm{Mo}_{1-x} \mathrm{Ru}_{x}\right)-(1-x)\left[E^{\mathrm{bcc}}(\mathrm{Mo})-E^{\mathrm{hcp}}(\mathrm{Mo})\right] .
\end{aligned}
$$

Usually, structural energy differences (or lattice stability) between first-principles calculations and CALPHAD show quite good agreement. However, for some transition elements, the disagreement between the two approaches is quite significant. ${ }^{35}$ Mo is one such case, with the structural energy difference between bcc and hcp from first-principles calculations and the CALPHAD approach differing by over $30 \mathrm{~kJ} / \mathrm{mol}$. After a rather extensive analysis, Kissavos et al. ${ }^{7}$ arrived at the conclusion that in order to reproduce enthalpy values close enough to the available experimental data ${ }^{36}$ the CALPHAD lattice stability $(11.55 \mathrm{~kJ} / \mathrm{mol})$ needed to be used for the value of the bcc $\rightarrow$ hcp promotion energy.

The SQS and CPA calculations are compared with the experimental measurements in Fig. 6. On the assumption that the experimental measurements by ${ }^{36}$ are correct, the derived enthalpy of formation of the hcp Mo-Ru system from the first-principles calculated lattice stability with the SQS and CPA approach in Fig. 6(a) cannot reproduce the experimental observation at all since the first-principles bcc $\rightarrow$ hcp lattice stability for Mo is $42 \mathrm{~kJ} / \mathrm{mol}$. Given this lattice stability, the only way in which the first-principles calculations within 

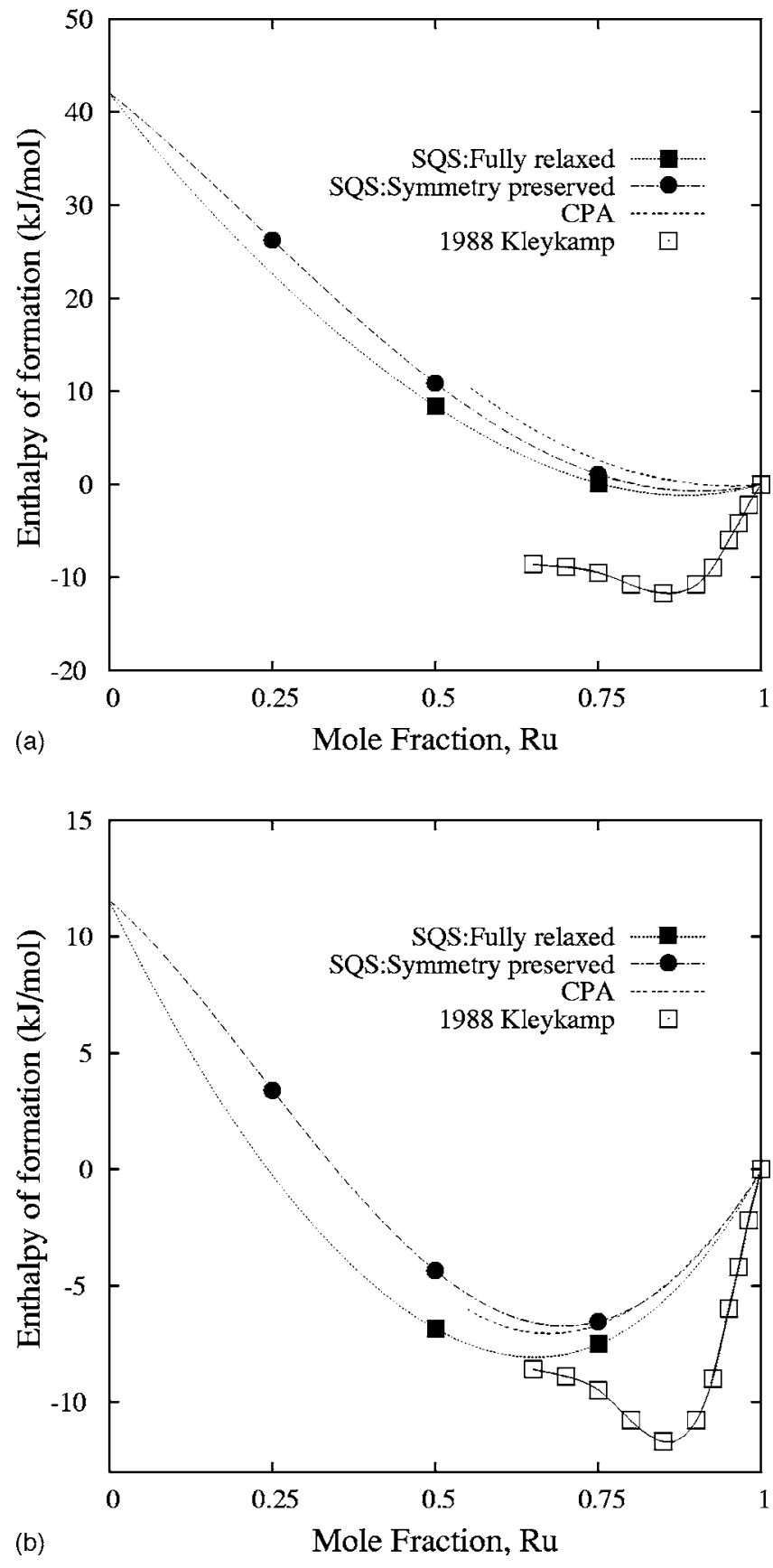

FIG. 6. Enthalpy of formation of the Mo-Ru system with both first principles and CALPHAD lattice stabilities. Reference states are bcc for Mo and hcp for Ru. (a) Enthalpy of formation of hcp phase in the Mo-Ru system from SQS's (this work) and CPA (Ref. 7). Total energy of hcp Mo is obtained from first-principles calculations in both cases. (b) Enthalpy of formation of hcp phase in the Mo-Ru system from SQS's and CPA. Total energy of hcp Mo is derived from the SGTE (Scientific Group Thermodata Europe) lattice stability.

both the SQS and CPA approaches would match the experimental results would be for the calculated enthalpy of mixing to be very negative, which is not the case. In fact, as can be seen in Fig. 6(a), the SQS and CPA calculations are very close to each other.
On the other hand, the enthalpy of formation derived from the CALPHAD lattice stability in Fig. 6(b) shows a better agreement than that from the first-principles lattice stability. It is important to note that the CALPHAD lattice stability was obtained through the extrapolation of phase boundaries in phase diagrams with Mo and stable hcp elements and, therefore, are empirical. The reason why such an empirical approach would yield a much better agreement with experimental data is still the source of intense debate within the CALPHAD community and has not been resolved as of now. The main conclusion of this section, however, is that the SQS's were able to reproduce the thermodynamic properties of hcp alloys as good as or better than the CPA method while at the same time allowing for the ion positions to locally relax around their equilibrium positions.

\section{G. IVA transition metal alloys}

The group IVA transition metals $\mathrm{Ti}, \mathrm{Zr}$, and Hf have hep structure at low temperatures and transform to bcc at higher temperatures due to the effects of anharmonic vibrations. When they form a binary system with each other, they show complete solubility for both the hcp and bcc solutions without forming any intermetallic compound phases in the middle.

The Hf-Ti binary is reported to have a low temperature miscibility gap and was modeled with a positive enthalpy of mixing by Bittermann and Rogl. ${ }^{37}$ Figure 7(a) shows remarkable agreement between the fully relaxed first-principles calculations and the thermodynamic model, which was obtained by fitting the experimental phase boundary data. Despite the fact that the local relaxation energies are rather large $(\sim 4 \mathrm{~kJ} / \mathrm{mol})$, the lattice parameters in both cases agree between each other and with the experimental results. ${ }^{39-41}$

In the case of the Ti-Zr binary, although no lowtemperature miscibility gap has been reported, Kumar et al. ${ }^{38}$ found that the enthalpy of mixing for the hep solutions in this binary was positive through fitting of phase diagram data. Our results confirm this finding, although with even more positive enthalpy. They are in fact similar in value to those calculated in the Hf-Ti alloys, suggesting that a low temperature miscibility gap may also be present in this binary.

In the Hf-Zr system no miscibility gap has been reported. The hep phase was modeled as an ideal solution $\left(\Delta H_{\text {mix }}\right.$ $=0)$ in the CALPHAD assessment. ${ }^{42}$ The present calculations suggest that the enthalpy of mixing of this system is positive, although rather small. In this case, it is expected that any miscibility gap would only occur at very low temperatures.

The three systems described in this section are chemically very similar, having the same number of electrons in the $d$ bands. Electronic effects due to changes in the widths and shapes of the DOS of the $d$ bands are not expected to be significant in determining the alloying energetics. Charge transfer effects are also expected to be negligible. The enthalpy observed can then be explained by just considering the atomic size mismatch between the different elements. As was shown in Table V, the Hf-Zr hcp alloys are the ones with 

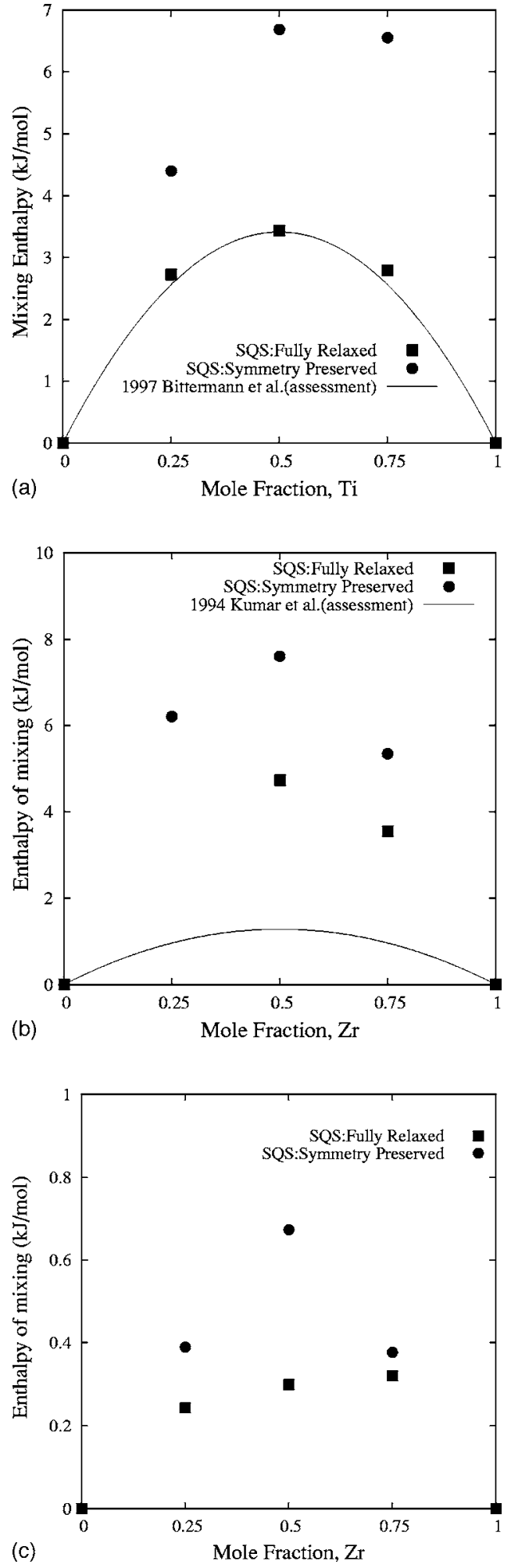

FIG. 7. Enthalpy of mixing for the Hf-Ti, Hf-Zr, and Ti-Zr binary hcp solutions calculated from first-principles calculations and CALPHAD thermodynamic models. All the reference states are hcp structures. (a) Calculated enthalpy of mixing for the hop phase in the Hf-Ti system compared with a previous assessment (Ref. 37). (b) Calculated enthalpy of mixing for the hcp phase in the Ti-Zr system compared with a previous assessment (Ref. 38). (c) Calculated enthalpy of mixing for the hcp phase in the Hf- $\mathrm{Zr}$ system. $\Delta H_{\text {mix }} \simeq 0$.
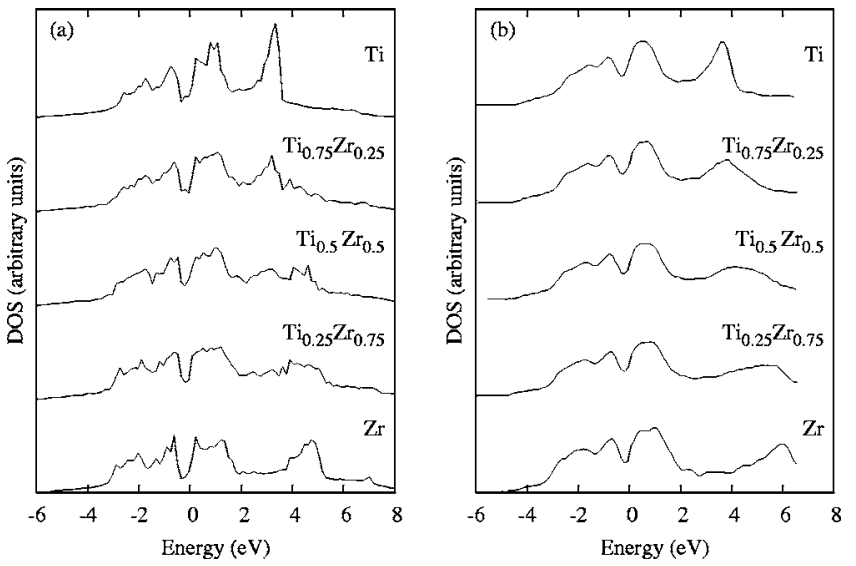

FIG. 8. Calculated DOS of $\mathrm{Ti}_{1-x} \mathrm{Zr}_{x}$ hcp solid solutions from (a) SQS and (b) CPA (Ref. 43).

the smallest difference in their lattice parameter, thus explaining their very small positive enthalpy of mixing.

As a final analysis of the ability of the generated SQS to reproduce the properties of random hcp alloys, Fig. 8 shows the alloying effects on the electronic DOS in Ti-Zr hcp alloys. The figure also presents the results obtained through the CPA approach by Kudrnovsky et al. ${ }^{43}$ As can be seen in the figure, both calculations predict that the DOS corresponding to the occupied $d$ states are virtually insensitive to alloying. The overall shape of the $d$-DOS remains relatively invariant. Since $\mathrm{Ti}$ and $\mathrm{Zr}$ have the same number of valence electrons, the fermi level remains essentially unchanged as the concentration varies from pure $\mathrm{Zr}$ to pure Ti. On the other hand, alloying effects are more pronounced in the $d$-DOS corresponding to the unoccupied states. Figure 8 shows how the broad peak at $\sim 4.5 \mathrm{eV}$ of the $d$-DOS for $\mathrm{Zr}$ is gradually transformed into a narrow peak at $\sim 3.0 \mathrm{eV}$ as the Ti content in the alloy is increased. The results from the CPA and the first-principles SQS calculations thus agree with each other, confirming the present results.

\section{SUMMARY}

We have created periodic special quasirandom structures with 16 atoms for binary hep substitutional alloys at three different compositions 25,50 , and 75 at. $\%$, to mimic the pair and multisite correlations of random solutions. The generated SQS's were tested in seven different binaries and showed fairly good agreement with existing experimental either enthalpy of mixing and/or CALPHAD assessments and lattice parameters. Analysis of the radial distribution and bond lengths in the 21 calculated SQS's, yielded a detailed account of the local relaxations in the hcp solutions and has been proven a useful way of characterizing the degree relaxation over several coordination shells.

It should also be noted that when using enthalpy of mixing to derive formation enthalpy to compare with experimental measurements, there can be a severe discrepancy between theoretical calculations and experimental data when the lattice stability, or structural energy difference, from firstprinciples calculation is problematic such as the Mo- $\mathrm{Ru}$ 
system in this work. This problem remains as an unsolved issue.

These supercells can be applied directly to any substitutional binary alloys to investigate the mixing behavior of random hep solutions via first-principles calculations without creating new potentials, as in the coherent potential approximation (CPA) or calculating other structures in the cluster expansion. Although the size of the current SQS's is not large enough to generate a supercell which can satisfy its correlation function at more than just three compositions ( $x$ $=0.25,0.5$, and 0.75 in $A_{1-x} B_{x}$ binary), calculations for these compositions can yield valuable information about the overall behavior of the alloys.

\section{ACKNOWLEDGMENTS}

This work is funded by the National Science Foundation (NSF) through Grant No. DMR-0205232. First-principles calculations were carried out on the LION clusters at the Pennsylvania State University supported in part by the NSF grants (DMR-9983532, DMR-0122638, and DMR-0205232) and in part by the Materials Simulation Center and the Graduate Education and Research Services at the Pennsylvania State University. We would also like to thank Christopher Wolverton at Ford for critical proofreading of the manuscript. Earle Ryba is acknowledged for his valuable advice for radial distribution analysis.
*Electronic address: dus136@psu.edu

${ }^{1}$ L. Kaufman and H. Bernstein, Computer Calculation of Phase Diagram (Academic Press, New York, 1970).

${ }^{2}$ N. Saunders and A. P. Miodownik, CALPHAD (Calculation of Phase Diagrams): A Comprehensive Guide (Pergamon, Oxford, 1998).

${ }^{3}$ A. van de Walle, M. Asta, and G. Ceder, CALPHAD: Comput. Coupling Phase Diagrams Thermochem. 26, 539 (2002).

${ }^{4}$ W. Kohn and L. J. Sham, Phys. Rev. 140, A1133 (1965).

${ }^{5}$ C. Wolverton, X. Y. Yan, R. Vijayaraghavan, and V. Ozolins, Acta Mater. 50, 2187 (2002)

${ }^{6}$ P. Soven, Phys. Rev. 156, 809 (1967).

${ }^{7}$ A. Kissavos, S. Shallcross, V. Meded, L. Kaufman, and I. Abrikosov, CALPHAD: Comput. Coupling Phase Diagrams Thermochem. 29, 17 (2005).

${ }^{8}$ Z. W. Lu, S. H. Wei, and A. Zunger, Phys. Rev. B 44, 3387 (1991).

${ }^{9}$ J. M. Sanchez, Phys. Rev. B 48, 14013 (1993).

${ }^{10}$ A. Zunger, S. H. Wei, L. G. Ferreira, and J. E. Bernard, Phys. Rev. Lett. 65, 353 (1990).

${ }^{11}$ S. H. Wei, L. G. Ferreira, J. E. Bernard, and A. Zunger, Phys. Rev. B 42, 9622 (1990).

${ }^{12}$ C. Jiang, C. Wolverton, J. Sofo, L.-Q. Chen, and Z.-K. Liu, Phys. Rev. B 69, 214202 (2004).

${ }^{13} \mathrm{G}$. Inden and W. Pitsch, in Phase Transformations in Materials, Vol. 5 of Materials Science and Technology: A Comprehensive Treatment (VCH, Weinheim, 1991), pp. 497-552.

${ }^{14}$ I. A. Abrikosov, S. I. Simak, B. Johansson, A. V. Ruban, and H. L. Skriver, Phys. Rev. B 56, 9319 (1997).

${ }^{15}$ G. Kresse and J. Furthmuller, Comput. Mater. Sci. 6, 15 (1996).

${ }^{16}$ G. Kresse and D. Joubert, Phys. Rev. B 59, 1758 (1999).

${ }^{17}$ J. P. Perdew, J. A. Chevary, S. H. Vosko, K. A. Jackson, M. R. Pederson, D. J. Singh, and C. Fiolhais, Phys. Rev. B 46, 6671 (1992).

${ }^{18}$ A. L. Spek, J. Appl. Crystallogr. 36, 7 (2003).

${ }^{19}$ A. A. Nayeb-Hashemi and J. B. Clark, Bull. Alloy Phase Diagrams 6, 246 (1985).

${ }^{20}$ M. Asta, R. McCormack, and D. de Fontaine, Phys. Rev. B 48, 748 (1993)

${ }^{21}$ R. R. Hultgren, Wiley Series on the Science and Technology of Materials (Wiley, New York, 1963).
${ }^{22}$ W. Hume-Rothery and G. V. Raynor, Proc. R. Soc. London, Ser. A 174, 471 (1940).

${ }^{23}$ F. W. Von Batchelder and R. F. Raeuchle, Phys. Rev. 105, 59 (1957).

${ }^{24}$ D. Hardie and R. N. Parkins, Philos. Mag. 4, 815 (1959).

${ }^{25}$ M. Hämäläinen, N. Bochvar, L. L. Rokhlin, and K. Zeng, J. Alloys Compd. 285, 162 (1999).

${ }^{26}$ R. Arroyave, D. Shin, and Z.-K. Liu, CALPHAD: Comput. Coupling Phase Diagrams Thermochem. 25, 230 (2005).

${ }^{27}$ H. Liang, S. L. Chen, and Y. A. Chang, Metall. Mater. Trans. A 28, 1725 (1997)

${ }^{28}$ I. Ansara, A. T. Dinsdale, and M. H. Rand, Vol. 2 of COST 507: Definition of Thermochemical and Thermophysical Properties to Provide a Database for the Development of New Light Alloys (European Cooperation in the Field of Scientific and Technical Research, Luxembourg, 1998).

${ }^{29}$ Y. Zhong, M. Yang, and Z.-K. Liu, CALPHAD: Comput. Coupling Phase Diagrams Thermochem. 29, 303 (2005).

${ }^{30}$ H. L. Luo, C. C. Chao, and P. Duwez, Trans. Metall. Soc. AIME 230, 1488 (1964).

${ }^{31}$ W. Hume-Rothery and G. V. Raynor, Proc. R. Soc. London, Ser. A 177, 27 (1941).

${ }^{32}$ G. V. Raynor, Proc. R. Soc. London, Ser. A 180, 107 (1942).

${ }^{33}$ R. S. Busk, Trans. Am. Inst. Min., Metall. Pet. Eng. 188, 1460 (1950).

${ }^{34}$ L. G. Wang, M. Sob, and Z. Zhang, J. Phys. Chem. Solids 64, 863 (2003).

${ }^{35}$ Y. Wang, S. Curtarolo, C. Jiang, R. Arroyave, T. Wang, G. Ceder, L.-Q. Chen, and Z.-K. Liu, CALPHAD: Comput. Coupling Phase Diagrams Thermochem. 28, 79 (2004).

${ }^{36}$ H. Kleykamp, J. Less-Common Met. 144, 79 (1988).

${ }^{37}$ H. Bittermann and P. Rogl, J. Phase Equilib. 18, 24 (1997).

${ }^{38}$ K. C. H. Kumar, P. Wollants, and L. Delaey, J. Alloys Compd. 206, 121 (1994).

${ }^{39}$ M. A. Tylkina, A. I. Pekarev, and E. M. Savitskii, Zh. Neorg. Khim. 4, 2320 (1959).

${ }^{40}$ Y. A. Chang (unpublished).

${ }^{41}$ E. Rudy (unpublished).

${ }^{42}$ H. Bittermann and P. Rogl, J. Phase Equilib. 23, 218 (2002).

${ }^{43}$ J. Kudrnovsky, V. Drchal, M. Sob, and O. Jepsen, Phys. Rev. B 43, 4622 (1991). 